Europhysics Letters

PREPRINT

\title{
Chaotic and power law states in the Portevin-Le Chatelier effect
}

\author{
M.S. Bharathi ${ }^{1}$ and G. Ananthakrishna ${ }^{1,2}$ \\ 1 Materials Research Centre, Indian Institute of Science, Bangalore-560012, India \\ 2 Centre for Condensed Matter Theory, Indian Institute of Science, Bangalore-560012, \\ India
}

PACS. 62.20.Fe - Deformation and plasticity.

PACS. 05.65.+b - Self-organized systems.

PACS. 05.45.Ac - Low-dimensional chaos.

\begin{abstract}
Recent studies on the Portevin - Le Chatelier effect report an intriguing crossover phenomenon from a low dimensional chaotic to an infinite dimensional scale invariant power law regime in experiments on $\mathrm{CuAl}$ single crystals and $\mathrm{AlMg}$ polycrystals, as a function of strain rate. We devise a fully dynamical model which reproduces these results. At low and medium strain rates, the model is chaotic with the structure of the attractor resembling the reconstructed experimental attractor. At high strain rates, power law statistics for the magnitudes and durations of the stress drops emerge as in experiments and concomitantly, the largest Lyapunov exponent is zero.
\end{abstract}

The Portevin-Le Chatelier (PLC) effect, discovered at the turn of the last century [1], is a striking example where collective behaviour of defects leads to complex spatio-temporal patterns 2, 3, 4. The PLC effect manifests itself as a series of serrations on the stress-strain curves when samples of dilute alloys are deformed under constant strain rate, $\dot{\epsilon}_{a}$ (actually constant pulling speed). The effect is observed only in a window of strain rates and temperatures. Each stress drop is associated with the formation and often the propagation of a dislocation band. In polycrystals, at low $\dot{\epsilon}_{a}$, the randomly nucleated type $\mathrm{C}$ bands with large stress drop amplitudes are seen. At intermediate strain rates, one finds the spatially correlated 'hopping' type $\mathrm{B}$ bands moving in a relay race manner with smaller stress drop amplitudes. At high strain rates, propagating type A bands with small amplitudes are observed. (In single crystals such a clear classification does not exist.) These different types of PLC bands are believed to represent distinct correlated states of dislocations in the bands. It is this rich spatio-temporal dynamics that has recently attracted the attention of physicists as well [8. 97. Indeed, the PLC effect is a good example of slow-fast dynamics commonly found in many stick-slip systems such as frictional sliding [5], fault dynamics [6] and peeling of an adhesive tape [7].

Recent efforts have shown that surprisingly large body of information about the nature of dynamical correlations is hidden in the stress-strain curves 10, 11, 12, 13]. More recently, an intriguing crossover phenomenon from a chaotic regime occurring at low and medium strain rates to a power law regime at high strain rates has been detected in experiments on the PLC

(c) EDP Sciences 
effect in $\mathrm{Cu}-10 \% \mathrm{Al}$ single crystals [12] and $\mathrm{Al}-\mathrm{Mg}$ polycrystals [13. This suggests that the crossover itself is insensitive to the microstructure. The purpose of this paper is to extend a model for the PLC effect introduced earlier [14 to explain this crossover phenomenon.

This crossover phenomenon is of interest in the larger context of dynamical systems as this is a rare example of a transition between two dynamically distinct states. Chaotic systems are characterised by the self similarity of the strange attractor and sensitivity to initial conditions quantified by fractal dimension and the existence of a positive Lyapunov exponent, respectively. In contrast, a class of spatially extended dissipative systems often exhibit a tendency to evolve to a marginally stable state, characterised by power law statistics for the events, under the action of slow external drive with no parameter tuning. (It must be emphasized that power laws can arise due to other mechanisms also [15].) Such a state is termed as selforganised criticality (SOC) by Bak et al [16]. Unlike the low dimensional nature of the chaotic attractor, SOC state is an infinite dimensional state. Large number of physical systems are known to exhibit SOC type features [17, 18].

There are numerous models and experiments where either of these dynamical regimes have been detected [19, 17, 18]. To the best of our knowledge ref. [12] is one of the two instances known where both these states are observed in one and the same system. The other example is in hydrodynamics where chaos is observed at low Rayleigh number and power law scaling regime, known as hard turbulence, is seen at high values [20]. Moreover, both the hard turbulence in convection and the power law regime in the PLC effect are observed at high drive parameter values in contrast to most SOC systems [17, 18]. Thus, power laws in the PLC effect are closer to the turbulent regime in convection than other SOC systems.

The microscopic origin of the PLC effect is due to the interaction of mobile dislocations with diffusing solute atoms and is referred to as dynamic strain aging (DSA), first suggested by Cottrell [4] and later improved by others. (See for instance ref. [2, 3]). At low strain rates (or high temperatures) the average velocity of dislocations is low, there is sufficient time for solute atoms to diffuse to dislocations and pin them (usually called as aging). Thus, longer the dislocations are arrested, larger will be the stress required to unpin them. When these dislocations are unpinned, they move at large speeds till they are pinned again. At high strain rates (or low temperatures), the time available for solute atoms to diffuse to the dislocations decreases and hence the stress required to unpin them decreases. Thus, in a range of strain rates and temperatures where these two time scales are typically of the same order of magnitude, the PLC instability manifests. The competition between the slow rate of pinning and sudden unpinning of the dislocations, at the macroscopic level translates into a negative strain rate sensitivity (SRS) of the flow stress as a function of strain rate which is the basic instability mechanism used in most phenomenological models [2, 3].

The well separated time scales implied in the DSA is mimicked by the fast mobile, immobile and the intermediate 'decorated' Cottrell type dislocations in the dynamical model due to Ananthakrishna and coworkers [14]. The basic idea of the model is that qualitative features of the PLC effect emerge from the nonlinear interaction of these few dislocation populations, assumed to represent the collective degrees of freedom of the system. The rate equations for dislocation densities are constructed based on known dislocation mechanisms. In spite of the idealised nature of the model, it is successful in explaining several generic features of the PLC effect, such as the emergence of the negative SRS 14,21, the existence of critical strain for the onset of the PLC instability, and the existence of a window of strain rates and temperatures for the occurrence of the PLC effect (see [14]). One prediction of the model is that there is a range of strain rates where the PLC effect is chaotic [22], subsequently verified by analysing experimental signals 110,11, 12, 13. The model has been studied in detail by our group and others including an extension to the case of fatigue [23, 24, 25]. 
Since the model is fully dynamical and it predicts chaos at intermediate strain rates found in experiments, it has the right ingredients for studying this crossover. Here, we outline the model in terms of scaled variables in the notation of Ref. [26]. In addition, we introduce a spatial coupling arising out of the cross-slip mechanism, used earlier by others [2, 3]. The model consists of densities of mobile, immobile, and Cottrell's type dislocations denoted by $\rho_{m}(x, t), \rho_{i m}(x, t)$ and $\rho_{c}(x, t)$ respectively, in the scaled form. The evolution equations are:

$$
\begin{aligned}
\frac{\partial \rho_{m}}{\partial t} & =-b_{0} \rho_{m}^{2}-\rho_{m} \rho_{i m}+\rho_{i m}-a \rho_{m}+\phi_{e f f}^{m} \rho_{m}+\frac{D}{\rho_{i m}} \frac{\partial^{2}\left(\phi_{e f f}^{m}(x) \rho_{m}\right)}{\partial x^{2}} \\
\frac{\partial \rho_{i m}}{\partial t} & =b_{0}\left(b_{0} \rho_{m}^{2}-\rho_{m} \rho_{i m}-\rho_{i m}+a \rho_{c}\right) \\
\frac{\partial \rho_{c}}{\partial t} & =c\left(\rho_{m}-\rho_{c}\right) .
\end{aligned}
$$

The first term in eqn.(1) refers to the formation of locks and consequent immobilisation of two mobile dislocations, the second term to the annihilation of a mobile dislocation with an immobile one, and the third term to the remobilisation of the immobile dislocation due to stress or thermal activation. The fourth term represents the immobilisation of mobile dislocations due to solute atoms. Once a mobile dislocation starts acquiring solute atoms we regard it as the Cottrell's type dislocation $\rho_{c}$. As they progressively acquire more solute atoms, they eventually stop, then they are considered as immobile dislocations $\rho_{i m}$ (loss term in eqn. (3) and the gain term in eqn. (2)). Alternately, the aggregation of solute atoms can be regarded as the definition of $\rho_{c}$, ie., $\rho_{c}=\int_{-\infty}^{t} d t^{\prime} \rho_{m}\left(t^{\prime}\right) K\left(t-t^{\prime}\right)$. For the sake of simplicity, we use a single time scale with $K(t)=e^{-c t}$. The convoluted nature of the integral physically implies that the mobile dislocations to which solute atoms aggregate earlier will be aged more than those which acquire solute atoms later ( see ref. [21]). The fifth term represents the rate of multiplication of dislocations due to cross-slip. This depends on the velocity of the mobile dislocations taken to be $V_{m}(\phi)=\phi_{\text {eff }}^{m}$, where $\phi_{\text {eff }}=\left(\phi-h \rho_{i m}^{1 / 2}\right)$ is the scaled effective stress, $\phi$ the scaled stress, $m$ the velocity exponent and $h$ a work hardening parameter. The last term is a spatial coupling term arising out of the nonlocal nature of the cross-slip as argued below. Actually, the nature of the spatial coupling in the PLC effect has been a matter of debate [2, 3. Within the scope of the model, a natural source of spatial coupling is the nonlocal nature of cross-slip as dislocations generated at a point spread over to the neighbouring elements. (Compatibility stresses between the slipped and the unslipped regions and long range interactions are other possible sources of coupling [2, 3].) Let $\Delta x$ be an elementary length. Then, the flux $\Phi(x)$ flowing from $x \pm \Delta x$ and out of $x$ is given by $\Phi(x)+\frac{p}{2}[\Phi(x+\Delta x)-2 \Phi(x)+\Phi(x-\Delta x)]$ where $\Phi(x)=\rho_{m}(x) V_{m}(x)$. Here $p$ is the probability of cross-slip spreading into neighbouring elements. Expanding $\Phi(x \pm \Delta x)$ and keeping the leading terms, we get $\rho_{m} V_{m}+\frac{p}{2} \frac{\partial^{2}\left(\rho_{m} V_{m}\right)}{\partial x^{2}}(\Delta x)^{2}$. Further, cross-slip spreads only into regions of minimum back stress. Noting that the back stress is usually taken to result from the immobile dislocation density ahead of it, we use $\Delta x^{2}=<\Delta x^{2}>=\bar{r}^{2} \rho_{\text {im }}^{-1}$. Here $\langle\ldots\rangle$ refers to the ensemble average and $\bar{r}^{2}$ is an elementary (dimensionless) length. Finally, $a, b_{0}$ and $c$ are the scaled rate constants referring, respectively, to the concentration of solute atoms slowing down the mobile dislocations, the thermal and athermal reactivation of immobile dislocations, and the rate at which solute atoms are gathering around the mobile dislocations. We note that the order of magnitudes of the constants have been identified in ref. 14, 23. These equations are coupled to the machine equation

$$
\frac{d \phi(t)}{d t}=d\left[\dot{\epsilon}-\frac{1}{l} \int_{0}^{l} \rho_{m}(x, t) \phi_{e f f}^{m}(x, t) d x\right]
$$


where $\dot{\epsilon}$ is the scaled applied strain rate, $d$ the scaled effective modulus of the machine and the sample, and $l$ the dimensionless length of the sample. (We reserve $\dot{\epsilon}_{a}$ for the unscaled strain rate.)

The PLC state is reached through a Hopf bifurcation. The domain of instability in $\dot{\epsilon}$ is $10<\dot{\epsilon}<2000$, and that in other parameters is the same as in the original model, beyond which uniform steady states exist . Here, we use $a=0.8, b_{0}=0.0005, c=0.08, d=0.00006, m=$ $3.0, h=0.07$ and $D=0.5$. (The parameter values used here are essentially the same used in several of our earlier calculations. [26].) But the results discussed below hold true for a wide range of other parameters in instability domain including a range of values of $D$.

These equations are discretised into $M$ equal parts of width $\Delta l$ and solved numerically for $\rho_{m}(j, t), \rho_{i m}(j, t), \rho_{c}(j, t), j=1,2, \ldots, M$, and $\phi(t)$. Due to the widely differing time scales, appropriate care has been exercised in the numerical solutions of these equations by using a variable step fourth order Runge-Kutta scheme with an accuracy of $10^{-4}$ for all the four variables. The spatial derivative of $\rho_{m}$ is approximated by its central difference. The initial values of dislocation densities are taken to be their steady state values with a Gaussian spread along the length of the sample with a Gaussian spread along the length of the sample, since the long term evolution of the system is essentially independent of the initial values. Now consider the boundary conditions. Since the sample is strained at the grips, we choose $\rho_{i m}(j, t)$ at $j=1$ and $N$ to be three orders of magnitude more than rest of the sample and $\rho_{m}(j, t)=\rho_{c}(j, t)=0$ at $j=1$ and $N$, as $\rho_{m}$ and $\rho_{c}$ cannot evolve into the grips .

We first recall the relevant experimental results on the crossover phenomenon and compare them with those from the model. Plots of two experimental stress-strain curves corresponding to the chaotic and SOC regimes of applied strain rates are shown in Fig. 1. In ref. [12], the chaotic nature of the stress-strain curve shown in Fig. 1a, was demonstrated by showing the existence of a finite correlation dimension using the standard Grassberger-Procaccia algorithm [27], and the existence of a positive Lyapunov exponent [19]. Both these methods involve embedding the time series in a higher dimensional space using time-delay technique [19]. (In addition, surrogate data analysis was also carried out in [12].) The correlation dimension, $\nu$, of the experimental attractor was found to be 2.3. Then, the number of degrees of freedom required for the description of the dynamics of the system is given by the minimum integer larger than $\nu+1$ which is four in this case, consistent with that used in the original model. The geometrical interpretation of these degrees of freedom is that it is the subspace to which the trajectories are confined. This dimension can also be obtained by an alternate method, called the singular value decomposition [28], which has an additional advantage of allowing the visualisation of the strange attractor. This method has been applied to the PLC time series earlier [11]. In this method, the trajectory matrix is constructed and the eigen values of the covariance matrix are calculated. For the time series in Fig. 1a, we find that the relative strength of the fourth eigen value drops more than two orders of magnitude compared to the first and changes very little beyond the fourth eigen value. Thus, we estimate the dimension of the experimental attractor to be four, consistent with that obtained from the correlation dimension. Using the first three principal directions of the subspace $C_{i} ; i=1$ to 3 , we have reconstructed the experimental attractor in the space of specifically chosen directions $C_{1}-C_{2}, C_{3}$ and $C_{1}$ to permit comparison with the model. This is shown in Fig. $2 \mathrm{a}$ for the experimental time series at $\dot{\epsilon}_{a}=1.7 \times 10^{-5} \mathrm{~s}^{-1}$. This can be compared with the strange attractor obtained from the model in the space of $\rho_{m}, \rho_{i m}$ and $\rho_{c}$ (at an arbitrary spatial location, here $j=50$ and $N=100$ ) shown in Fig. $2 \mathrm{~b}$ for $\dot{\epsilon}=120$ corresponding to the mid chaotic region (see below). Note the similarity with the experimental attractor particularly about the linear portion in the phase space (Fig. 2a) identified with the loading direction in Fig. 1a. Note that the identificaton of the loading direction is consistent with the absence 
of growth of $\rho_{m}$. Since the existence of a positive Lyapunov exponent is a confirmatory test of chaos, we have calculated both the largest Lyapunov exponent (LLE) and the spectrum of Lyapunov exponents [19] directly from the model. Since the magnitude of the LLE and that of the largest Lyapunov exponent calculated from the spectrum agree, we have shown the average LLE (obtained over 15000 time steps after stabilisation) in Fig. 3a as a function of the strain rate for $N=100$. The LLE becomes positive around $\dot{\epsilon}=35$ reaching a maximum at $\dot{\epsilon}=120$, and practically vanishing around 250. (Periodic states are observed in the interval $10<\dot{\epsilon}<35$.) For $\dot{\epsilon} \geq 250$, the dispersion in the value of the LLE is $\sim 5 \times 10^{-4}$ which is the same order as the mean. Thus, the LLE is taken to vanish beyond $\dot{\epsilon}=250$ as is expected of the power law regime [29].

At high strain rates beyond $\dot{\epsilon} \sim 280$, the stress-time series obtained from the model shows no inherent scale in the magnitudes of the stress drops as in the case of experimental time series at high strain rates 12, 13]. We have analysed the distributions for the stress drop magnitudes $\Delta \phi$ and their durations $\Delta t$. The distribution of stress drop magnitudes, $D(\Delta \phi)$, shows a power law $D(\Delta \phi) \sim \Delta \phi^{-\alpha}$. This is shown in Fig. 3b(०) along with the experimental points $(\bullet)$ corresponding to $\dot{\epsilon}_{a}=8.3 \times 10^{-5} \mathrm{~s}^{-1}$. Clearly both experimental and theoretical points show a scaling behaviour with an exponent value $\alpha \approx 1.1$. The distribution of the durations of the drops $D(\Delta t) \sim \Delta t^{-\beta}$ also shows a power law with an exponent value $\beta \approx 1.3$. The conditional average of $\Delta \phi$ denoted by $\langle\Delta \phi\rangle_{c}$ for a given value of $\Delta t$ behaves as $\langle\Delta \phi\rangle_{c} \sim \Delta t^{1 / x}$ with $x \approx 0.65$. The exponent values satisfy the scaling relation $\alpha=x(\beta-1)+1$ quite well. Since the basic cause of the stress-drops is the growth of mobile dislocation density during this period, one can look at the scaling behaviour of the total density in the sample at a given time using $\bar{\rho}_{m}(t)=\int \rho_{m}(x, t) d x$. Let $\Delta \bar{\rho}_{m}(t)$ denote the increase in $\bar{\rho}_{m}(t)$ occurring during the intervals of the stress drops. Then, one should expect that the statistics of $\Delta \bar{\rho}_{m}$ also to exhibit a power law, ie., $D\left(\Delta \bar{\rho}_{m}\right) \sim \Delta \bar{\rho}_{m}^{-\gamma}$, with the same exponent value as $\alpha$. A plot of $D\left(\Delta \bar{\rho}_{m}\right)$ for $N=300$ is shown in Fig. $3 \mathrm{~b}(\diamond)$. The extent of the power law regime is nearly two orders with $\gamma \approx 1.1$, same as $\alpha$. The large bump at high values is due to the effect of finite size of the system as in many models [6] and in particular, here it is due to high levels of stress at the grips. Noting that dislocation bands cannot propagate into regions of high stresses, it is clear that the edges cause distortions in the otherwise smoothly propagating bands leading to large changes in $\bar{\rho}_{m}(t)$. Typically, the influence of the edges are felt by the band when it is 20 sites away. Increasing $N$ from 100 to 300, increases the scaling regime by half a decade and the peak of the bump reduces from 700 to 500, thus indicating the influence of the finite size of the system.

To sum up, the present model exhibits chaotic dynamics at low and medium strain rates, and SOC dynamics at high $\dot{\epsilon}$. These distinct dynamical states and the crossover clearly emerge due to the inclusion of nonlinearities in the form of basic dislocation mechanism and a spatial coupling. As for the nature of dynamics in different regions of strain rate, one can perhaps anticipate the emergence of the spatio-temporal chaotic regime [30, as the original model exhibits chaos. However, the emergence of SOC dynamics needs some explanation. Recently, we have shown that the upper limit of the PLC effect in the original model is a result of a reverse Hopf bifurcation at high strain rates [26]. This implies that the average amplitude of the stress drops decreases as a function of strain rate as in experiments. This means that the average stress level (in time) is roughly constant with small fluctuations around the mean implying that $\dot{\epsilon}$ essentially balances the total plastic strain rate. The picture essentially remains unaltered when spatial coupling is introduced. Thus, in this regime, the state of the system is critically poised as in any SOC system. Recently, we have shown that the geometry of the slow manifold has a bent structure and the 'fold line' on the slow manifold corresponds to the threshold value of stress for unpinning the dislocations. (See figs. 4 and 5 in ref. [21] 
EUROPHYSICS LETTERS

and also for further details.) Interestingly, for the SOC regime, we find that most of the spatial elements are literally on the 'fold line' corresponding to the marginally stable state.

The dynamical origin of the scaling regime in our model is similar to that dealt by Gil and Sornette [31] using a subcritical Hopf bifurcation. More recently, a dynamical analysis of Zhang's model of SOC has been reported as well [32]. However, it must be pointed out that in the present case, the scaling occurs at high drive values in contrast to many SOC type models. In this sense, the dynamical regimes found in our model are similar to that observed in thermal convection of a box of helium gas, namely, periodic states $\rightarrow$ chaos $\rightarrow$ power law hard turbulence regime [20] as a function of Rayleigh number. (Actually, chaos and hard turbulence are separated by soft turbulence.) Lastly, to the best of our knowledge, this is the first model which exhibits such a crossover not just in the context of the PLC effect, but as a general crossover between two distinct dynamical regimes.

$* * *$

We thank Prof. Neuhäuser for supplying the experimental data. This work is supported by Department of Science and Technology, Grant No: SP/S2/K-26/98, New Delhi, India.

\section{REFERENCES}

[1] Le Chatelier F., Rev. de Métallurgie, 6 (1909) 914; Portevin A. and Le Chatelier F., C.R. Acad. Sci. Paris, 176 (1923) 507.

[2] Viewpoint Set No 21, Propagative Instabilities. Scripta. Metall., 29 (1993) 1147.

[3] Kubin L.P., Fressengeas C. and Ananthakrishna G., Collective Behaviour of Dislocations, edited by Nabarro F.R.N., Dislocations in Solids, Vol. 11 (North-Holland, Amsterdam) 2002.

[4] Cottrell A.H., Dislocations and Plastic Flow in Crystals (University Press, Oxford) 1953.

[5] Persson B.N.J. and Tosatti E., Physics of Sliding Friction (Kluwer Academic Publishers, Dordrecht) 1996.

[6] Carlson J.M. And Langer J.S., Phys. Rev. Lett., 62 (1989) 2632; Phys. Rev. A., 40 (1989) 6470.

[7] Maugis D. and Barquins M., Adhesion, edited by Allen K.W., Vol. 12 (Elsevier, London) 1988, p. 205.

[8] D'Anna G. and Nori F., Phys. Rev. Lett., 85 (2000) 4096.

[9] Franklin S.V., Mertens F. and Marder M., Phys. Rev. E., 62 (2000) 8195.

[10] Ananthakrishna G., et al.,, Scripta. Metall., 32 (1995) 1731.

[11] Noronha S.J., et al., Int. J. of Bifurcation and Chaos, 7 (1997) 2577.

[12] Ananthakrishna G., et al., Phys. Rev.E., 60 (1999) 5455.

[13] Bharathi M.S., et al., Phys. Rev. Lett., 87 (2001) 165508.

[14] Ananthakrishna G. and Valsakumar M.C., J. Phys. D., 15 (1982) L171.

[15] See for instance chapter 14 of Sornette D., Critical Phenomena of Natural Sciences: Chaos, Fractals, Self-Organization and Disorder: Concepts and Tools (Springer, Heidelberg) 2000.

[16] Bak P., Tang C. and Wiesenfeld K., Phys. Rev. Lett., 59 (1987) 381 ;Phys. Rev. A., 38 (1988) 364.

[17] Jensen H.J., Self-Organised Criticality (Cambridge University Press, Cambridge) 1998.

[18] BAK P., How Nature Works (Springer - Verlag, NewYork) 1996.

[19] Abarbanel H.D.I., Analysis of Observed Chaotic Data (Springer-Verlag, New York) 1996.

[20] Heslot F., Castaing B. and Libchaber A., Phys. Rev. A., 36 (1987) 5870.

[21] Rajesh S. and Ananthakrishna G., Phys. Rev. E., 61 (2000) 3664.

[22] Ananthakrishna G. and Valsakumar M.C., Phys. Lett., A95 (1983) 69.

[23] Bekele M. and Ananthakrishna G.,Phys. Rev. E., 56 (1997) 6917.

[24] Glazov M.V., et al., Appl. Phys. A, 64 (1997) 373. 
[25] Zaiser M., et al., Comp. Mater. Science, 5 (1999) 35.

[26] Rajesh S. and Ananthakrishna G., Physica D, 140 (2000) 193.

[27] P. Grassberger and I. Procaccia, Physica D, 9 (1983) 189.

[28] Broomhead D. and King G., Physica D, 20 (1987) 217.

[29] Chen K., Bak P. and Obukhov S.P.,Phys. Rev. A., 43 (1991) 625.

[30] See Cross M.C. and Hohenberg P.C., Rev. Mod. Phys., 65 (1993) 851 for a discussion on the transition from chaos to spatio-temporal chaos.

[31] Gil L. and Sornette D., Phys. Rev. Lett., 96 (1996) 3991.

[32] Blanchard Ph., Cessac B. and Krüger T., J. Stat. Phys., 88 (1997) 307; J. Stat. Phys., 98 (2000) 375; Cessac B., Blanchard Ph. and Krüger T., Phys. Rev. E., 64 (2001) 016133.
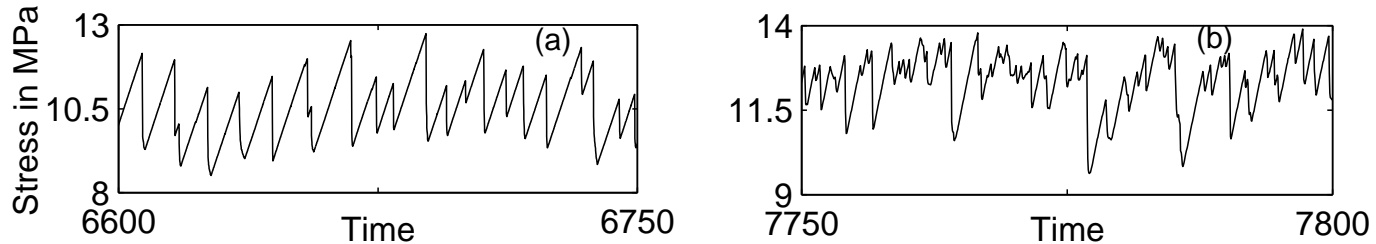

Fig. 1 - Experimental stress-time series corresponding to (a) chaotic state at $\dot{\epsilon}_{a}=1.7 \times 10^{-5} \mathrm{~s}^{-1}$ and (b) SOC state at $\dot{\epsilon}_{a}=8.3 \times 10^{-5} \mathrm{~s}^{-1}$.
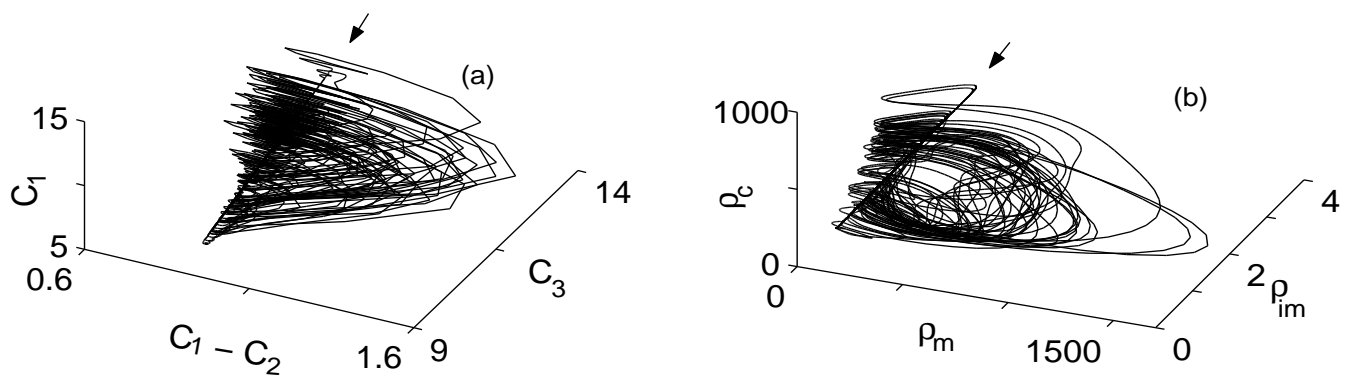

Fig. 2 - (a) Reconstructed experimental attractor (b) Attractor from the model for $N=100, j=50$.
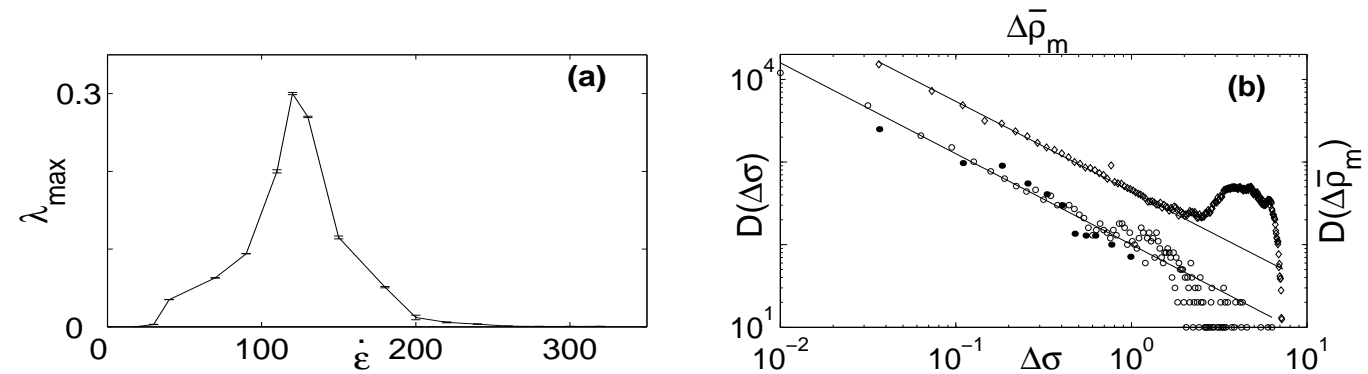

Fig. 3 - (a). The largest Lyapunov exponent of the model. (b) Distributions of the stress drops from the model $(\circ)$, from experiments $(\bullet)$ and $\Delta \bar{\rho}_{m}(\diamond)$ from the model. Solid lines are guide to the eye. 